\title{
Observations sur l'usage linguistique des poilus pendant la Grande Guerre et sa mise en scène rétrospective dans les bandes dessinées
}

\author{
Sybille Große / Lena Sowada
}

Universität Heidelberg

Abstract

Dieser Beitrag untersucht den künstlerischen sprachlichen Ausdruck in drei ausgewählten Comics, die sich etwa 100 Jahre nach dem Ersten Weltkrieg mit diesem auseinandersetzen. Dazu wird die ästhetisierte Sprache dem authentischen Sprachgebrauch einfacher Soldaten, sog. Poilus, in privater Korrespondenz und Kriegstagebüchern, die zwischen 1914 und 1918 verfasst wurden, gegenübergestellt. Aus einer auf den Wortschatz gerichteten Perspektive sind die authentischen und die künstlerischen Texte durch eine Affinität zum populär- und umgangssprachlichen Französisch sowie, in geringerem Maße, zum argot des tranchées geprägt. Die in den Comics inszenierte und als fingiert charakterisierte Mündlichkeit zeichnet sich im Vergleich zu den authentischen Texten durch einen spielerischeren Sprachgebrauch und eine stärkere Präsenz von Vulgarismen aus. Weiterhin trägt der stilisierte und fiktionale Charakter der Sprache in den Comics zu einer ausgeprägteren Variation populär- und umgangssprachlicher sowie dem Argot zugehöriger Lexeme bei, insbesondere bei der Bezeichnung des deutschen Feindes, die in diesem Maße in den Texten der zeitgenössischen Soldaten nicht zu finden sind.

Dans cette contribution, nous étudions l'expression linguistique artistique dans une sélection de trois bandes dessinées sur la Première Guerre mondiale qui furent publiées environ cent ans après la fin de la guerre. Pour ce faire, nous confronterons ce langage stylisé à l'usage linguistique authentique de poilus tel qu'il apparaît dans leur correspondance privée et dans les carnets de guerre. Sous l'angle du lexique, les textes authentiques et les textes artistiques se caractérisent par une même affinité avec le français populaire et familier et, dans une moindre mesure, avec l'argot des tranchées. L'oralité mise en scène dans les BD et caractérisée comme oralité simulée se distingue en revanche par un emploi plus ludique de la langue et par un nombre plus élevé de vulgarismes par rapport aux documents authentiques. De plus, le caractère fictif et stylisé des BD contribue à une variation plus prononcée de lexèmes argotiques, populaires et familiers, notamment dans la désignation de l'ennemi allemand, que nous ne trouvons pas chez les scripteurs soldats contemporains de la Première Guerre mondiale.

In this contribution, we analyse the artistic linguistic expression in three selected comic strips on the First World War that were published about 100 years after the end of the war. Therefore, we compare the aestheticized language with the authentic linguistic usage of ordinary people in their private correspondence and in war diaries written between 1914 and 1918. From a lexical point of view, both authentic and artistic texts are marked by an affinity with popular and colloquial French and, to a lesser extent, with trench slang (argot des tranchées). Orality portrayed in comics and identified as simulated orality is characterized by a more playful use of language and a higher number of vulgarisms compared to the authentic documents. In addition, the fictional and stylized character of the comics contributes to a more pronounced variation of slang, popular and familiar lexemes, especially in the designation of the German enemy, which we do not find in the texts of contemporary soldiers.

\section{Keywords}

historische Soziolinguistik • Poilus • Comics • fingierte Mündlichkeit

sociolinguistique historique • poilus • bandes dessinées • oralité simulée

historical sociolinguistics $\bullet$ poilus $\bullet$ comics $\bullet$ simulated orality

\section{INTRODUCTION}

\subsection{Introduction générale et description des corpus d'analyse}

La Première Guerre mondiale est un évènement communicatif (Guilhaumou 1996) car elle réunit des soldats issus de diverses régions et d'un statut social varié, usant de langages différents. Elle constitue également le moment déclencheur d'une augmentation massive de la production écrite (Mercier 2015). La séparation des familles provoquée par la mobilisation de milliers de soldats amène la majeure partie de la 
population française à écrire pour maintenir le contact avec les proches. Ce développement n'a pas d'exclusivité sociale particulière : même les personnes ayant peu l'habitude d'écrire s'expriment par ce médium dans des lettres, des cartes postales ou des carnets de guerre. Ces documents authentiques nous permettent d'accéder, au moins en partie, à l'usage linguistique d'une majorité démographique (Elspaß 2012 : 161) au début du XXe siècle.

Notre contribution portera sur l'usage linguistique des poilus ordinaires pendant la Première Guerre mondiale sous deux angles complémentaires : nous exposerons d'abord brièvement quelques traits saillants du lexique tel qu'il apparaît dans des documents authentiques. Ensuite, nous proposerons de confronter cet usage authentique à l'usage artistique de la langue mise en scène dans des bandes dessinées (BD) publiées rétrospectivement sur la thématique de la « Grande Guerre » pour savoir comment le langage des poilus est présenté et par quels moyens l'expression artistique vise à évoquer ce langage environ cent ans après la fin de la guerre ${ }^{1}$. Par conséquent, notre étude s'appuiera sur deux corpus différents : le premier comprend des documents authentiques issue de la correspondance privée de soldats ordinaires dont la plus grande partie est issue de l'est de la France, notamment des Vosges et de l'Alsace ${ }^{2}$. Le deuxième corpus réunit une sélection de bandes dessinées mettant en scène la Première Guerre mondiale dans une optique rétrospective.

Sur cette toile de fond seront analysées de façon qualitative trois BD sur la Grande Guerre :

- Putain de guerre ! 1914-1918 (Tardi / Verney 2014),

- Ambulance 13 (Mounier / Cothias / Ordas 2010),

- Les poilus. 1. Frisent le burn-out (Bouzard 2016).

Les trois BD du corpus se distinguent nettement au niveau de la mise en forme ainsi qu'au niveau du contenu. Tandis que la BD de Tardi / Verney (2014) prend en compte la guerre dans toute sa durée, en racontant son évolution et ses atrocités dans la perspective de simples soldats, les deux autres BD se concentrent sur la guerre à deux moments précis. Ambulance 13 décrit ainsi les premières vingt-quatre heures d'action d'un jeune médecin après son arrivée au front en 1916. Pour tous, poilus, infirmiers et infirmières, la guerre est dépourvue de sens. La seule définition valable pour caractériser la vie dans les tranchées et sur le front est l'idée d'une boucherie sanglante. La BD de Bouzard (2016) $)^{3}$ est la plus humoristique

\footnotetext{
Des études linguistiques qui mettent au centre de leur analyse des textes littéraires afin de pouvoir décrire l'usage populaire du français, donc également des gens ordinaires, ne sont pas rares (voir François 1985), mais elles ne sont pas sans problèmes méthodologiques parmi lesquels la « fréquente monotonie de la représentation » figure au premier plan (Gadet $1992: 13$ )

2 Concernant les correspondances privées et journaux intimes consultés, voir le proje Egoling14-18 : https://heidelberger-forum-edition.de/egoling14-18 (dernière consultation 1/12/2020). Depuis 2014, plusieurs études ont été consacrées à différents aspects de l'usage linguistique des auteurs de ces documents dont il est impossible de décrire icil'ensemble ge linguistique des auteurs de ces documents dont il est impossible de décrire icil 'ensemble des resultats. A titre d'exemple, voir Sowada (2018) pour le langage de l'immédiat communicatif, Große / Sowada (202

3 Dans cette BD sont intégrées également les " lettres à Susanne " que les poilus écrivent pour un camarade décédé. Le fait que la correspondance soit traitée ainsi dans les
} BD au niveau du contenu met en avant son importance pour les acteurs du conflit. des trois, mais elle est, comme les deux autres, clairement une BD anti-guerre.

La BD chronologique de Tardi / Verney (2014) est plus didactisée et, contrairement aux deux autres, sa dimension textuelle se limite à des récits sans passages dialogiques et sans phylactères. Le texte est encadré par des citations d'hommes politiques contemporains et enrichi par une description chronologique des faits historiques par Jean-Pierre Verney, historien et spécialiste de la Première Guerre mondiale. Afin de permettre la compréhension du vocabulaire des tranchées, la BD est complétée par un glossaire.

\subsection{La guerre comme sujet dans les BD}

Les premières bandes dessinées sur la guerre sont publiées dans les années 1930, d'abord dans des journaux anglophones, puis en tant que publications indépendantes, comme Terry and the pirates de Sickles / Caniff en 1934 (Rifas 2016 : 183-184). Les BD de guerre deviennent un genre populaire, surtout pour la Seconde Guerre mondiale (Borcherding / Mertins / Riemer 2018 : 8). C'est pourquoi les années 1930 et 1940 sont considérées comme l'âge d'or de la BD de guerre (Rifas : 185). La représentation réaliste de la guerre fait son entrée plus tard, durant la guerre de Corée (ibid. : 186). À partir des années 1970, les sujets tels que la guerre, la déportation ou les dictatures sont fréquemment repris dans les bandes dessinées (BaronCarvais 2007 : 116-118).

En France, les BD deviennent un médium courant pour le traitement des sujets historiques, souvent avec des propos pédagogiques et spécialement pour un public jeune (Krieger / Machnik / Schmitz 2018 : 59). La Première Guerre mondiale est bien présente dans des BD françaises, notamment avec l'œuvre de Jacques Tardi, un des auteurs de bande dessinée les plus influents (Bocklage / Otto 2018 : $25)$; et le centenaire de la Grande Guerre contribue à l'appropriation de ce conflit par les BD.

\subsection{L’argot des tranchées: le langage de la guerre}

Au cours de la guerre, l'idée de la naissance d'un nouveau langage dans les tranchées circule dans la société française, propagée d'abord par les journaux et plus tard par la littérature. Les soldats contemporains eux-mêmes se montrent souvent sceptiques ou bien critiques (Steuckardt 2018 : 28-29), car ils se méfient des exagérations de la presse, des idées déformées sur la vie au front circulant dans la population et de l'instrumentalisation propagandiste de la langue dite « poilue » (Dauzat $1919: 24-25)^{4}$

Cet argot n'est pas un nouveau sociolecte qui se forme pendant la Première Guerre mondiale, car l'argot militaire est déjà en usage dans les casernes bien avant 1914. D'ailleurs, il ne s'agit pas d'une variété homogène, mais d'une variété

4 La méfiance des soldats envers cette nouvelle dénomination est également reprise par Tardi / Verney (2014 : 30) : «On s'est rendu compte qu'on dérangeait les civelots avec nos puanteurs et notre crasse. On nous appelait 'poilus' ». 
qui intègre des lexèmes de différents argots et qui présente des affinités avec d'autres registres et sociolectes :

Le parler des Poilus n'est pas un argot né de la guerre, mais un millefeuille fait de diverses strates : langage populaire avec quelques traces d'argot parisien, argot militaire et argot né dans les tranchées, français « ordinaire » de tous les jours, des villes et des campagnes (Rézeau 2018 : 11).

L'argot utilisé par les poilus puise principalement dans le langage populaire parisien, dans le langage des casernes en France et en Algérie et, dans une moindre mesure, dans certains dialectes. Environ un tiers des termes caractérisés par leur appartenance à l'argot des tranchées est constitué de néologismes (Dauzat 1919 : 7 ; Déchelette [1918] 1972 : 8) car, comme tout conflit belligérant, la Première Guerre mondiale constitue un moment d'innovation lexicale :

Une secousse aussi formidable, bouleversant aussi profondément et aussi longtemps la vie contemporaine, ne pouvait manquer de provoquer des répercussions sur l'instrument de la pensée (Dauzat 1919 : 9).

Les lexèmes argotiques répondent à un besoin d'expressivité élevée et marquent nettement une prise de distance par rapport à la réalité, par exemple à travers une langue métaphorique ou humoristique. Ils font participer l'interlocuteur à l'arrière à la vie quotidienne des soldats. Dans une moindre mesure, ces lexèmes comblent des lacunes sémantiques (Bianchi 2015 : 138).

\section{L'USAGE LINGUISTIQUE AUTHENTIQUE}

L'usage linguistique dans les correspondances et les carnets rédigés par des scripteurs peu habitués à écrire offre un reflet de la langue parlée au quotidien dans le médium écrit. En communiquant avec leurs proches par écrit, les scripteurs se servent, en règle générale, de la variété dont ils se serviraient dans une situation de communication similaire en coprésence d'interlocuteurs ${ }^{5}$. Cette variété se rapproche, à plusieurs égards, de la langue de l'immédiat communicatif : elle comprend des influences du langage populaire, de la langue familière et des traits régionaux. Ces affinités sont surtout perceptibles au niveau du lexique, mais on les trouve également dans des structures morphosyntaxiques empruntées à l'immédiat communicatif6 (Große et al. 2016 : 7 ; Koch / Oesterreicher 2011 : 12 ; Mahrer $2017: 17$ ).

- Ceci ne signifie pas que les textes soient dénués d'éléments relevant de la distance communicative ou appartenant au type de texte écrit qu'est la lettre. C'est surtout l'emploi de formules épistolaires qui est dû à la communication écrite.

6 Le terme de l'immédiat communicatif est introduit par Koch et Oesterreicher (1986 2011) dans un modèle pour concevoir les différences entre l'oralité et la scripturalité qui se sont avérées trop simplistes pour rendre compte de la variété des conceptions textuelles. Les textes proches de l'immédiat communicatif sont typiques des situations de commun cation informelles, privées et intimes, caractérisées de plus par un haut degré d'implication émotionnelle et personnelle des interlocuteurs. L'immédiat communicatif au sens large comprend les sociolectes, les registres populaires, familiers ou argotiques et aussi des parlers régionaux (Koch / Oesterreicher 2011: 17)
Le français familier est la variété utilisée dans des situations de communication en famille, entre amis et avec des proches, caractérisée par une ambiance informelle et une proximité (mentale) personnelle. II n'est pas propre à un groupe précis de locuteurs et il peut se rapprocher du français populaire ou du français courant selon le locuteur. Traditionnellement, le français familier est associé au médium oral (PrüßmannZemper 1990 : 838 ; Müller 1975 : 204-205).

Le français populaire est également proche de la langue orale (François 1985 : 298), mais se distingue du simple français familier par une connotation sociale moins soutenue. Au début du $X X^{e}$ siècle, le français populaire surgit à Paris comme « le langage du ( peuple >, du ( petit peuple >, voire du ‘ bas peuple > » (François 1985 : 296). Quant aux parlers régionaux ${ }^{7}$ et aux argots, le français populaire assume un rôle de relais en popularisant des mots issus de ces variétés marquées dans le français courant (Müller 1975 : 203-204).

\section{LES PARTICULARITÉS DE LA «MISE EN MOTS » ARTISTIQUE DANS LES BD : LA SPÉCIFICITÉ DU LANGAGE VERBAL ET VISUEL}

Si les bandes dessinées sont d'habitude facilement identifiées en tant que telles, leur définition analytique s'avère plus compliquée. Une caractéristique essentielle est leur séquentialité (Eisner 1985 : 147) : les images composent une séquence délibérée et consciemment organisée, intégrant diverses disciplines telles que la psychologie, l'histoire, l'anatomie ou bien les sciences du langage. C'est ainsi que la BD transmet des informations et produit une sensation esthétique chez le lecteur (McCloud 1993 : 9) .

La mixité typique entre images et paroles requerrait une approche "plurisémiotique » (Giaufret 2013) ou multimodale (Cohn 2012) car cette combinaison d'images et de paroles forme un langage différent qui est qualifié par Cohn (2012) de « visual language » :

This leads structured sequential sounds to be spoken languages of the world, structured sequential body motions become sign languages, and structured sequential images literally become visual languages (Cohn 2012 : 6).

Les textes des BD, et par conséquent l'expression linguistique, peuvent prendre des formes variées selon Giaufret (2013), Pietrini (2007: 47-48) et Quinquis (2004: 13-14), ce qui situe la parole à différents niveaux énonciatifs :

7 Pour nous, les 'parlers régionaux' comprennent plus qu'un lexique spécifique à une région, qui est d'ailleurs très difficile à identifier définitivement et en exclusivité pour un espace donné (cf. aussi Tuaillon $1978 \cdot 152$ ). Souvent les lexèmes ne sont pas propres a espace donne (cf. aussi Tuaillon 1978 : 152). Souvent les lexemes ne sont pas propres a une seule localité ou région, mais ils sont aussi en usage dans des zones plus vastes. De plus, il ne s'agit pas toujours de lexèmes particuliers, mais d'une signification régionalement divergente d'un lexème tout à fait courant. Néanmoins, les caractéristiques régionales ne se limitent pas à un lexique particulier, elles apparaissent également au niveau phonétique ou morphosyntaxique ou encore pragmatique. II est très difficile d'identifier si un élément correspond au français régional d'une aire plus ou moins vaste, à un substrat dialectal ou bien à une autre langue de contact.

8 Pour une discussion de différentes approches définitoires à la bande dessinée, cf. Meskin (2016) 
- les énoncés et pensées dans les phylactères

- les voix off

- les textes intégrés dans l'image (onomatopées, coupures de journaux etc.)

- les commentaires de l'éditeur (par ex. «fin de l'épisode »).

La partie textuelle la plus importante pour notre analyse concerne les dialogues exprimés dans les phylactères. Ils se caractérisent par les trois aspects fondamentaux suivants :

- Au niveau iconique, ils mêlent les images et les paroles.

- Au niveau linguistique, ils se distinguent par un usage diastratiquement marqué comme populaire ou familier.

- Au niveau de l'authenticité et de la fiction, ils sont considérés comme fictifs et non pas authentiques (voir aussi Glaude 2014).

La succession des phylactères imite d'habitude le changement des prises de parole (turn-taking) des différents acteurs et le caractère dialogique des conversations (Bramlett 2016 : 382). L'analyse du "parlé » dans les textes littéraires remonte à des études des romanistes allemands Goetsch (1985) ou Blank (1991) qui ont introduit le terme de fingierte Mündlichkeit pour désigner l'oralité simulée (Sinner $2014: 227)^{9}$. Ce terme s'avère utile pour l'analyse des éléments textuels-verbaux des BD, car le langage utilisé n'est pas le reflet fidèle de l'oral, mais l'emploi intentionnel de certains éléments isolés du langage de l'immédiat communicatif en faveur d'un effet stylistique (Hennig / Jacob 2016 : 191). L'oralité conceptuelle (Koch / Oesterreicher 2011) se manifeste sous des formes très diverses dans les textes littéraires (cf. Goetsch 1985). Parmi les différentes fonctions de l'oralité simulée dans la littérature, on pourrait citer les suivantes :

- créer l'illusion d'une langue de l'immédiat communicatif,

- renforcer l'illusion en se rapprochant du réel,

- situer l'action dans une région donnée ou/et par rapport à une période concrète,

- marquer l'appartenance sociale d'un personnage,

- caractériser les personnages (naïveté, stupidité, manque d'éducation, exotisme ou danger) et

- suggérer de l'authenticité dans la présentation des personnages ou bien évoquer un monde rustique en contraste avec le monde moderne (Fludernik 2011 : 33 ; Goetsch 1985 : 217).

Le caractère fictionnel et stylisé du texte se reconnaît grâce au choix de certaines variantes diastratiques, diaphasiques ou diatopiques mises en relief tandis que d'autres ne sont pas prises en compte (voir aussi Blanche-Benveniste 1997 : 103) : « Wichtig ist anzumerken, dass die Evozierung von Mündlichkeit in Erzähltexten nach dem Prinzip der selektiven

9 II y a une très grande variété d'approches de ce phénomène de l'oralité représentée dans des textes fictifs et, par conséquent, une énorme richesse de dénominations de ce terme (cf. aussi Affolter 2011). En romanistique, Thun (2005) utilise le terme de literarisierte Mündlichkeit et Schlieben-Lange (1983) celui de Semi-Oralität.
Überbetonung typischer Merkmale kolloquialer oder dialektaler Rede funktioniert » (Fludernik 2011 : 33).

De plus, étant donnée la nature écrite du médium et la représentation graphique de certains éléments marqués com-

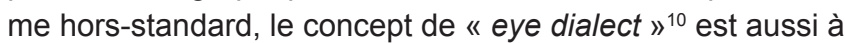
prendre en compte (Bramlett 2016 : 383) qui sert à caractériser linguistiquement les personnages et leur milieu socioculturel. La narration invite le lecteur à constituer le monde fictif dans l'acte de lecture. Pour ce faire, elle se base sur une situation de communication concrète en empruntant des éléments à l'oralité associée à la situation de communication (Goetsch 1985 : 218). C'est dans cette perspective qu'incombe au lecteur, en tant que récepteur de ces traits reproduits à l'écrit, un rôle important (Affolter 2011).

\section{L'ANALYSE DES USAGES LINGUISTIQUES AUTHENTIQUE ET ARTISTIQUE}

\subsection{Résultats des analyses du corpus des correspon- dances des poilus (1914-1918)}

Pour notre analyse, nous partons de l'usage linguistique authentique dans les écrits des poilus ordinaires qui servira de fond et de repère pour la description du langage artistique stylisé dans les $\mathrm{BD}$. Les observations ci-dessous résultent de nos études antérieures, plus étendues et que nous allons ici centrer sur notre objectif d'analyse comparative.

Le lexique utilisé dans les lettres, les cartes postales et les carnets de guerre se caractérise par l'interaction de différentes variétés sociales, différents registres et dialectes, notamment du français familier, du français populaire, des parlers régionaux et de l'argot des tranchées ${ }^{11}$.

Notre analyse du lexique prend en considération la biographie individuelle des scripteurs ainsi que leur alphabétisation écrite et leurs habitudes scripturales présumées. L'éventail de registres dont dispose un scripteur donné est étroitement lié au degré de sa compétence scripturale générale et de son expérience dans la rédaction de textes. Plus un scripteur est expérimenté, plus son expression à l'écrit est riche et variée. Au niveau de l'argot des tranchées, c'est-à-dire l'argot des poilus, notre corpus des écrits privés et des correspondances présente surtout des formes argotiques expressives, telles que marmite « gros obus ennemi » (Déchelette 1972 [1918] : 134), cafard « ennui, mélancolie, idées noires » (ibid. : 53) ou crapouillot « lance-bombes, canon de tranchées » ou « projectile du crapouillot » (Dauzat 1919 : 256), ou bien des termes techniques militaires comme gourbi « abri du front, plus ou moins rudimentaire et plus ou moins spacieux, souvent enterré » (Rézeau 2018 : s.v. gourbi) ou guitoune « abri du front, plus ou moins rudimentaire, allant de la tente à une construction en dur » (ibid. : s.v. guitoune), deux emprunts

10 Bramlett (2016:383) définit le eye dialect qu'il qualifie de « strategic manipulation of spelling to approximate a character's pronunciation ».

11 L'attribution d'une forme linguistique donnée à un registre ou à une variété peut s'avérer compliquée vu le chevauchement des variétés, notamment du français populaire et familier ou du français populaire et de l'argot. Ce qui est décisif pour nous, c'est le marquage d'une forme linguistique et moins son identification avec un registre informel déterminé. 
à l'arabe (Rey 2010 : s.v. gourbi ; s.v. guitoune). Le lexème boche, désignation de l'ennemi, représente la moitié des occurrences argotiques dans le corpus et il est utilisé en tant que substantif (1), substantif générique (2) ou bien comme adjectif (3) avec des graphies variées :

(1) mais il sant bien plus dangéreu les maudie boche (Eugène Lorieau, 1915) ${ }^{12}$

(2) Oui ! nous avons fait reculé le Bôche et de beaucoup mais ça ne s'est pas fait tout seul. (Paul Grandemange, 13.9.1918)

(3) Nous avons creusé un boyau et élargi une tranchée boche (Albert Provot, 12.6.1915)

La haute fréquence de cette désignation de l'ennemi dans l'usage des scripteurs confirme l'usage courant de boche. En même temps, l'utilisation restreinte du vocabulaire argotique dans le corpus de textes authentiques ne correspond pas à ce que les études contemporaines sur l'argot des tranchées et les dictionnaires publiés pendant la Première Guerre mondiale et à la fin de celle-ci pourraient suggérer (cf. Dauzat 1919 ; Déchelette 1972 [1918] ; Sainéan 1915).

En outre, le lexique illustré dans la correspondance et les carnets de guerre contient des éléments familiers, courants dans le français quotidien tels que binette "tête ridicule " (Littré 1873-1874 : s.v. binette) ; "visage » (TLFi 1994 : s.v. binette) (4), caser « mettre à telle ou telle place, trouver une place pour quelque chose " (TLFi 1994 : s.v. caser) (5) ou gosse « enfant, adolescent » (ibid. : s.v. gosse) (6).

(4) je t'envoi ma petite binette (Aloïs Grandemange, 3.10.1914)

(5) dans la maison ou nous logeions il y avais 20 têtes à caser (Paul Labriet, 13.2.1916)

(6) celà nous fait rire comme des gosses (Albert Provot, 2.1.1915)

Le français familier se caractérise par un degré élevé de spontanéité, d'affectivité, d'émotion et d'emphase, ce qui résulte d'un emploi de lexèmes ayant une signification spécifique ou d'une tendance à former beaucoup de nouveaux mots (Müller 1975 : 205). La formation de substantifs avec le suffixe -ard, -arde est très fréquente dans le français familier (Paulikat 2017 : 60), dont débrouillard ${ }^{13}$ « qui sait se débrouiller ; habile à se tirer d'affaire et à parvenir à ses fins " (TLFi 1994 : s.v. débrouillard) est un exemple parmi d'autres :

(7) Et le principal c'est d'être débrouillard (Paul Grandemange, 16.10.1914)
Un autre procédé typique du français familier pour former des mots consiste à les raccourcir (Huot 2005 : 27 ; Müller 1975 : 207 ; Prüßmann-Zemper 1990 : 839). Il est entre autres illustré par sous-off pour sous-officier :

(8) quelques sous-off, adjudants seuls s'en occupent! (Émile Garnier, 4.8.1914)

Un exemple de structure phraséologique marquée comme familière est casser la croûte " manger amicalement et sans façon avec lui » (Littré 1873-1874, s.v. croûte) :

(9) Nous étions trois camarades des environs, comme nous nous étions approvisionné de pain, conserves etc, nous nous sommes dis il faut casser la croûte, car demain où nous serons (Justin Poinçot, 19.8.1914)

D'autres formes familières sont des interjections telles que dame (10) «Interjection explétive qui est une formule d'affirmation » (Littré 1873-1874 : s.v. dame) ou vivement (11) pour exprimer un souhait (TLFi 1994 : s.v. vivement).

(10) Tout de suite remis, on se met à causer de chose et d'autres et je lui ai payé une bon-ne goutte! Dame, il ne l'avait pas volé, car il avait un peu chaud, et ici il fait froid. (Joseph Grandemange jun., 13.11.1914)

(11) vivement qu'on rechasse tous ces cochons là de chez nous (Paul Labriet, 9.1.1916)

L'usage des formes hypocoristiques est également caractéristique du français familier et correspond à l'implication émotionnelle des interlocuteurs. Dans les lettres, ces formes sont fréquentes dans les parties qui désignent l'interlocuteur ou dans des passages d'autodénomination.

(12) Bons baisers à Chalo et fifine (Paul Grandemange, 19.5.1918)

L'emploi des hypocoristiques renforce l'affectivité de la lettre et en même temps les relations interpersonnelles à distance, ce qui est spécifique de la correspondance épistolaire.

Les formes lexicales appartenant au français populaire sont relativement peu fréquentes dans le corpus. Citons, à titre d'exemple, les verbes barder (13) " être mauvais, dur, dangereux » (Bauche 1920 : 191) ou ficher (14) « planter, mettre, poser ou faire » (Bauche $1920: 230)$ :

(13) pour le moment ça barde ou je suis. (Joseph Colle, 15.3.1916)

(14) tâche de plus toussé pour revenir sans cela en te fichera une distribution (Maria Saunier, 20.11.1916)

L'expressivité du registre populaire est illustrée par deux exemples tirés du corpus:

(15) Ça va leur donner à réfléchir à ces Salops. (Paul Grandemange, 20.7.1918)
12 Les textes du corpus des écrits privés ont été translitérés de façon diplomatique, c'està-dire en respectant les graphies, la ponctuation et la mise en page originale.

13 Si l'adjectif débrouillard peut avoir perdu sa marque " familière " dans le français du XXle siècle, ceci ne semble pas être le cas pour le début du XXe siècle. La première attesusage familier à cette époque-là (cf. TLFi 1994 : s.v. débrouillard). 
(16) St Maurice le 31 janvier 1916 Mon cher poteau Je viens repondre a la charmante lettre du 26 ct. (Paul Colle, 31.1.1916)

Le premier exemple montre la dénomination de l'ennemi (concrètement : les Allemands) et le deuxième la relation des épistoliers et l'affectivité qui règne entre eux. En comparaison avec les registres mentionnés, l'expression linguistique des scripteurs ne montre qu'une moindre influence régionale. Tout de même, on distingue la fréquence relativement élevée de la collocation avoir le temps long après qqn.

(17) Pauvre Joseph il doit avoir le temp long après ses frères (Joseph Grandemange sen., 16.1.1916)

(18) J'ai été heu-reux de recevoir de vos nouvelles; car je commençais à avoir le temps long aprés vous !.. (Aloïs Grandemange, 11.4.1918)

(19) J'ai le temps long de passer quelques bons moments auprès de vous mes Chers Parents (Paul Grandemange, 19.9.1918, lettre 1)

Cette collocation est usuelle dans le français parlé en Belgique avec la connotation "trouver le temps long", " s'ennuyer et se languir de qqn. ". Ces variantes sont de plus courantes dans l'est de la France, plus précisément dans les Ardennes et en Lorraine (Poirier / Francard 2019 : s.v. avoir le temps long). Rézeau (2018 : s.v. temps) atteste cette structure également pour le département du Haut-Rhin, Martin / Lienhard (1907 : s.v. Zit) même dans le dialecte alsacien germanique. La répartition régionale explique cet usage dans notre corpus, car les auteurs des extraits mentionnés sont issus des Vosges méridionales et de l'Alsace.

Dans tous les registres, le corpus montre que des lexèmes portant des marques diasystématiques peuvent être mis en relief par différentes stratégies typographiques. Parmi ces stratégies, nous pouvons énumérer le soulignement, l'usage des guillemets simples ou doubles, français ou allemands.

(20) si vous aviez vu cette "pagaille» (Paul Grandemange, 31.8.1918)

Cette mise en relief pourrait s'expliquer par la volonté des scripteurs de souligner la connotation spécifique d'un lexème usuel, de se distancier d'un lexème ou encore par la volonté de faire comprendre aussi aux interlocuteurs le quotidien linguistique du front en marquant le vocabulaire spécifique et peut-être non usuel pour ceux de l'arrière.

\subsection{La mise en scène artistique du parler des poilus}

Pour l'analyse linguistique des BD, nous proposons une approche qualitative de leurs particularités lexicales liées à l'expression artistique sans prétendre toutefois en donner un panorama complet. Les BD présentent une grande variété lexicale, notamment des lexèmes populaires, familiers et de l'argot des tranchées. En revanche, la variation régionale, elle aussi peu fréquente dans le corpus de lettres, y fait quasiment défaut. II faut souligner avec Goetsch (1985 : 202) que l'oralité dans les textes littéraires est toujours, en quelque sorte, fictive et qu'elle fait partie d'un style et d'une stratégie esthétique consciente de l'auteur.

Parmi le vocabulaire populaire utilisé dans les BD, nous repérons par exemple merlan (Tardi / Verney 2014 : 30) « coiffeur » (Bauche 1920 : 248), l'hosto (Tardi / Verney 2014 : 31 ; Mounier / Cothias / Ordas $2010: 5$ ) « hôpital » (Bauche 1920 : 240), bouffer (Tardi / Verney $2014: 34)$ " manger » (Bauche 1920 : 197) ou faire gaffe (Tardi / Verney 2014 : 33 ; Mounier / Cothias / Ordas 2010 : 33) « faire attention » (TLFi 1994 : s.v. gaffe). Parmi ces exemples, seul le dernier est également documenté dans notre corpus de lettres. Ceci montre qu'il peut y avoir un décalage entre l'attestation d'un lexème donné dans un dictionnaire (ce qui prouve sa lexicalisation et par conséquent son entrée dans la langue) et les fréquences réels d'usage. II s'agit ici, bien évidemment, des indications sans valeur représentative.

Le processus de formation de mots avec la suffixation en -ot et en -ard, très productive dans le langage populaire et familier en général, se retrouve par exemple dans civelots (Tardi / Verney 2014 : 30) « civil » (Bauche 1920 : 209), cuistots (Tardi / Verney 2014 : 30) « cuisinier " (Bauche 1920 : 216), veinard (Tardi / Verney $2014: 27$; Bouzard 2016 : 40) « celui qui a de la chance, d'heureuses veines » (Littré 1873-1874 : s.v. veinard) ou flambard (Tardi / Verney 2014 : 33) marqué comme familier et signifiant « gai luron, fanfaron, vaniteux " (TLFi 1994 : s.v. flambard). Le registre familier se manifeste aussi dans des verbes tels que débarquer (Bouzard $2016: 8$ ) " arriver à l'improviste " (TLFi 1994 : s.v. débarquer) ou ripailler (Bouzard $2016: 40$ ) " repas où l'on mange et où l'on boit avec excès » (TLFi 1994 : s.v. ripaille). Concernant le lexique marqué comme vulgaire, citons à titre d'exemple se foutre de qqn. (Bouzard 2016 : 26) « n'avoir cure de quelque chose, de quelqu'un ; n'en faire aucun cas » (TLFi 1994 : s.v. foutre) et déconner (Tardi / Verney $2014: 15)$ " dire ou faire des conneries » (TLFi 1994 : s.v. déconner).

Le lexique des BD présente donc des continuités par rapport au corpus de lettres authentiques dans la mesure où nous y retrouvons des lexèmes familiers, populaires ou argotiques souvent selon des procédés de formation de mots productifs. Ceci ne peut guère surprendre car beaucoup de scènes des BD reproduisent des situations de communication entre pairs, en famille ou entre amis, dans lesquelles les locuteurs utilisent d'habitude un langage relâché et moins soutenu.

En comparant les lexiques, il devient tout de même manifeste que le langage des BD contient un nombre beaucoup plus élevé de gros mots et de vulgarismes que la correspondance familiale. 
(21) Ça c'est une putain de bonne nouvelle de bite à couilles (Bouzard 2016 : 44)

(22) Son copain chiasseux (Tardi / Verney $2014: 28$ )

(23) C'est vrai qu'ils sont chiants (Bouzard $2016: 15)$

(24) C'est vraiment la merde... (ibid. : 18)

(25) Dans ta gueule les boches !!! (ibid. : 37)

La concentration d'expressions vulgaires est particulièrement forte dans la BD de Bouzard (2016) qui présente clairement l'approche la plus satirique et humoristique. L'emploi généralement plus élevé de vulgarismes dans les BD pourrait s'expliquer par le fait que les bandes dessinées représentent souvent des scènes entre soldats et sur le front, tandis qu'une lettre à l'épouse, par exemple, s'inscrit dans d'autres situations de communication que l'expérience immédiate des tranchées et des combats.

L'usage de l'argot des tranchées est aussi beaucoup plus développé dans les BD que dans notre corpus de textes authentiques. Un exemple attesté dans les deux corpus est marmite comme dans " un trio de 'marmites' explose » (Mounier / Cothias / Ordas 2010 : 6). Les scripteurs des lettres n'utilisent, au-delà de la dénomination boche, que quelques expressions relevant de l'argot des tranchées. En revanche, les BD présentent une variation de formes argotiques plus abondante.

En ce qui concerne la dénomination de l'ennemi allemand, les trois BD puisent dans l'ensemble des désignations suivantes: (voir tableau 1)

Sur ce plan, les BD de Bouzard (2016) et de Tardi / Verney (2014) sont très riches. Les auteurs utilisent pratiquement tout l'inventaire possible des dénominations pour les Allemands qui furent en usage pendant la guerre. Cette joie d'alterner les synonymes atteint un niveau presque " sur-ambitieux » et souligne en même temps l'aspect stylisé des BD. En ce qui concerne la dénomination de l'ennemi, les scripteurs contemporains utilisent surtout la désignation boche avec des qualifiants divers : sale boche, maudits boches, bandits de boches, vaches de boche.
Si l'on compare le langage mis en scène dans les BD et celui employé par les scripteurs de l'époque de la Première Guerre mondiale, le premier se caractérise par la fréquence plus élevée d'une grande variété d'expressions populaires, familières et argotiques. Quelques-unes semblent avoir intégré la langue française au moment même de la Première Guerre mondiale avec une lexicalisation plus tardive, comme par exemple feldgrau (1922 selon le TLFi) ${ }^{14}$.

L'emploi linguistique prémédité et artistique est aussi sensible dans des jeux de mots dans les BD:

(26) les pieds dans les champs de blé, la tête au champ d'honneur (Tardi / Verney 2014 : 5).

L'humour et le sarcasme caractérisent également les extraits suivants par rapport à l'enthousiasme initial « Pourtant on avait confiance. Dès la porte de Bagnolet, Berlin était tombé » (Tardi / Verney 2014 : 5), à la tenue militaire des soldats français « [...] et on pouvait dire sans se tromper qu'on n'était pas discrets dans la campagne, d'autant plus qu'avec nos costumes de cirque, on était des cibles épatantes " (ibid. 2014 : 13) ou bien encore par rapport à l'histoire coloniale des membres de l'Entente:

(27) Les Anglais tenaient à ce que les peuplades de leur empire colonial - qu'ils avaient 'éduquées' et auxquelles ils avaient apporté les bienfaits inestimables de leur magnifique civilisation - participent un peu à leur guerre, ne serait-ce que par décence, histoire de rendre un petit service à leurs 'bienfaiteurs'.... Que les choses n'aillent pas toujours dans le même sens ! (Tardi / Verney 2014 : 29).

Les BD prennent rétrospectivement position contre la guerre, ce qui est illustré, par exemple, par l'assimilation des

14 Le terme feldgrau est absent dans notre corpus de correspondances privées, mais il est attesté pour l'année 1915 dans le corpus de lettres de la Première Guerre mondiale établi récemment par Rézeau $(2018: 367)$ ce qui montre une fois de plus, que l'usage d'un étément donné dans une communauté linguistique précède son entrée dans les dictionnaires.

\begin{tabular}{|l|l|l|}
\hline Bouzard (2016) & Tardi / Verney (2014) & Mounier / Cothias / Ordas (2010) \\
\hline & les Allemands & \\
\hline les boches, sale boche, putain de boche & les boches, barbares boches, vandales boches & les boches \\
\hline les Alboches & les Alboches & Fritz \\
\hline Fritz & & les Frisés \\
\hline les Frisés & & \\
\hline les fridolins & & \\
\hline Allez le schleu & & \\
\hline & les pruscos, soldats prussiens & \\
\hline & un casque à pointe & \\
\hline & un feldgrau & \\
\hline & nos « ennemis héréditaires » & \\
\hline & les Teutons, une balle teutonne & \\
\hline & les Huns & \\
\hline
\end{tabular}

Tableau 1 : Désignations pour les Allemands dans les BD. 


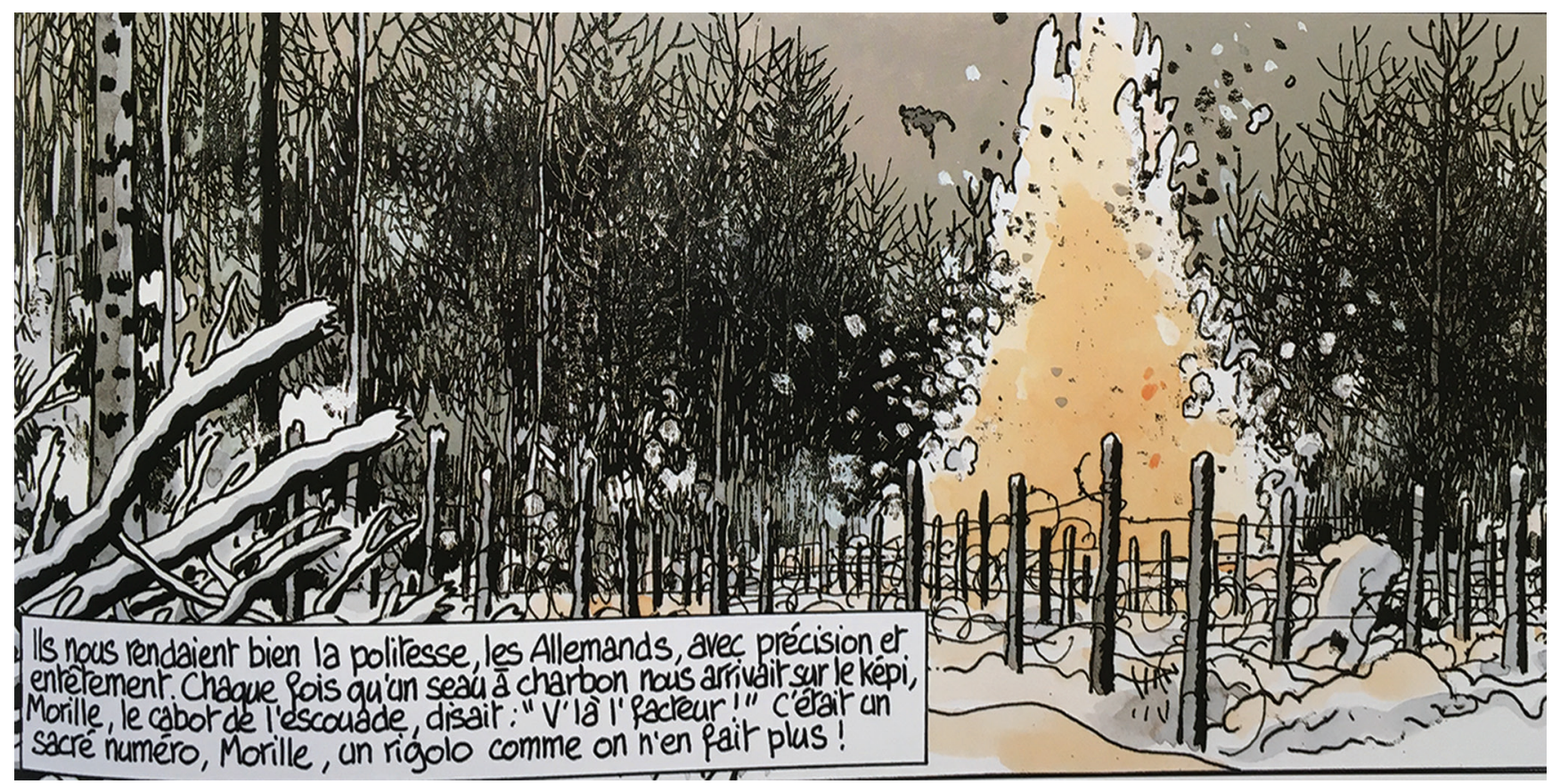

Image 1: Extrait de Putain de guerre ! 1914-1918, Tardi / Verney (2014 : 21). (C) Casterman. Avec l'aimable autorisation des auteurs et des Editions Casterman.

tranchées à des tombes (Tardi / Verney $2014: 20$ ), par le parallélisme entre le « sacrifice collectif librement consenti » avec la guerre (ibid. : 11) ou encore par la similarité des soldats allemands et français : " C'était la première fois qu'on pouvait reluquer de près nos ennemis... Ils nous ressemblaient, en somme » (ibid. : 7).

La stratégie de l'évocation visuelle d'une certaine variété parlée à travers le code écrit, qualifiée également de " eye dialect », apparaît dans les trois exemples suivants :

(28) Le capiston avait eu son compte, et bien qu'on soye habitués, c'était pas bon pour notre moral de voir dans quel état on l'avait mis (Tardi / Verney 2014 : 36)

(29) C'est pas beau l'progrès ? (Tardi / Verney $2014: 45$ )

(30) En arrivant, d'mandez I'major Garnier. C'est lui qui commande les s'cours, là-haut... Prenez vot' temps. Y z'enterrent vot' prédécesseur, le lieut'nant Jaquin... (Mounier / Cothias / Ordas $2010: 6$ )

II s'agit toujours du choix conscient de l'auteur de représenter certains éléments variationnels dans des textes littéraires. Selon Goetsch (1985 : 212), cette représentation n'est ni strictement mimétique ni entièrement artificielle. Elle reprend les éléments jugés caractéristiques pour évoquer une certaine réalité linguistique et communicative. Nous ne trouvons pas cette intégration consciente des reflets d'une prononciation dialectale dans les textes authentiques. Si nous en trouvons, il semble que ce soit dû à la situation de communication privée et, dans tous les cas, cela reste occasionnel.

Les caractéristiques de l'expression linguistique ne se définissent pas seulement au niveau du lexique, mais également au niveau de la structuration des phrases et de l'organisation de l'information. L'expression linguistique au quotidien, dans des situations de communication informelle, est marquée par différents aspects morphosyntaxiques de l'oralité conceptuelle. Une caractéristique bien attestée de la langue informelle est la négation postverbale en omettant la particule ne (Koch / Oesterreicher 2011 : 172-173 ; Blanche-Benveniste 1997 : 37). Cette caractéristique du français oral, même si elle y est attestée, ne se retrouve qu'avec une moindre fréquence dans le corpus de lettres. Dans le corpus des BD, elle semble par contre être utilisée à une fréquence plus élevée. Une autre caractéristique du français informel au niveau de la morphosyntaxe concerne l'omission du pronom-sujet dans des tournures impersonnelles telle qu'il y a ou il faut. Cette caractéristique morphosyntaxique présente également des affinités avec le langage populaire (Koch / Oesterreicher 2011 : 177). Les deux corpus attestent de tournures avec une omission du pronom personnel sujet, par exemple y a (31) ou faut (32) :

(31) Ya Bapt qui attend la relève de garde, les gars ! (Bouzard $2016: 5)^{15}$

(32) Allez, il recommence á neiger, faut pas trainer.. (Cothias / Ordas / Mounier 2010 : 33)

Notre corpus de lettres présente également des exemples d'omission du pronom-sujet : enfin vaut encore mieux cela que d'être tué (Paul Labriet, 20.3.1915) et comme les enfants sont ingrats suffit qu'on les choiyent ils ne pensent plus á leurs parents (Antoinette Perrin, 18.9.1915).

15 Cf. également la structure présentative typique du langage informel dans cet exemple. 
Nous concluons cette étude avec un extrait de Tardi / Verney (2014) qui présente une partie des caractéristiques mentionnées ci-dessus et qui en illustre bien la dimension ironique. Au niveau de l'organisation syntaxique, la dislocation à droite Ils nous rendaient bien la politesse, les Allemands est typique du langage de l'immédiat communicatif, mettant en avant une suite agrégative qui suit le fil des idées du locuteur selon lequel l'élément connu est introduit par un pronom et précisé après coup. Le lexique comprend cabot " caporal " (Dauzat 1919 : 249) relevant de l'argot des tranchées, sacré « à valeur intensive » du français familier (TLFi 1994 : s.v. sacré) ainsi que rigolo « qui amuse, fait rigoler » (ibid. : s.v. rigolo). De plus, l'énonciation en discours direct V'là l'facteur ! reprend l'idée d'un eye dialect en stylisant la prononciation à travers l'écrit.

\section{CONCLUSION}

A l'issue de cette étude évidemment trop fragmentaire et sur la base d'une documentation qui est loin d'être exhaustive, nous voudrions avancer quelques conclusions partielles. Nous constatons d'abord bel et bien un rapprochement entre l'usage linguistique des poilus dans leur correspondance et leur usage linguistique stylisé dans les BD. Dans l'expression artistique, les différents registres qui caractérisent également l'usage authentique sont utilisés en fonction d'une caractérisation sociale des protagonistes dans le contexte de la guerre. Dans cette perspective, les auteurs des BD se présentent comme bien renseignés sur les usages linguistiques des poilus, d'autant qu'ils distinguent parfaitement l'usage linguistique des soldats ordinaires de celui de leurs supérieurs hiérarchiques (les officiers, par exemple). La différence dans l'usage des éléments populaires, familiers ou bien argotiques réside dans le fait que les BD vont au-delà des écrits authentiques: nous pouvons identifier certains traits d'un langage presque " sophistiqué », reprenant et amplifiant des éléments de l'imaginaire culturel et linguistique lié à la Première Guerre mondiale. Par rapport aux BD, les tournures et mots relevant de l'argot des tranchées sont moins diversifiés et moins riches dans le corpus de lettres authentiques et ne se concentrent que sur quelques lexèmes.

Les caractéristiques d'un langage informel, lié à la situation de communication des soldats dans les tranchées et avec leurs familles, se retrouvent dans les deux corpus à des degrés divers et avec une importance variable.

La désignation de l'ennemi met en avant une différence entre les deux corpus car elle s'avère beaucoup moins variée dans les lettres, cartes postales et carnets de guerre que dans les BD. Dans ce contexte, il convient de souligner que les poilus, dans leurs correspondances, abordent notamment des sujets quotidiens et parlent rarement de la guerre et des acteurs impliqués. II s'agit ici clairement d'une stylisation rétrospective et d'une mise en œuvre du panorama linguistique dans les BD. Visant à authentifier la situation de communication, le vocabulaire des BD intensifie visiblement l'emploi du lexique utilisé par ces scripteurs.

Les bandes dessinées, avec leur prédilection à styliser l'usage linguistique, rejoignent les rangs des textes littéraires dans lesquels la mise au point verbale joue un rôle de premier plan. De plus, les BD se caractérisent par une mise en œuvre visuelle des émotions et des séquences d'action, cet aspect n'ayant pu cependant être pris en compte dans cette étude comparée qui, avant tout, a ciblé les éléments verbaux.

Pour conclure, nous aimerions souligner une autre continuité entre les lettres et les BD : dans les BD de Tardi / Verney (2014) et de Bouzard (2016), la rédaction et la réception des lettres sont bien présentes en tant qu'activités vitales pour les soldats et leurs familles, ce qui souligne parfaitement, de manière rétrospective, l'importance de la correspondance pour la survie émotionnelle et quotidienne des poilus.

\section{Bibliographie}

\subsection{Sources primaires}

Bouzard, Guillaume (2016), Les Poilus. 1. Frisent le burn-out, Paris, Audie-Fluide glacial (Les formidables aventures des poilus).

Cothias, Patrick / Ordas, Patrick / Mounier, Alain (2010), L'Ambulance

13. Tome 1 : Croix de sang, Charnay-lès-Mâcon, Bamboo.

Tardi, Jacques / Verney, Jean-Pierre (2014), Putain de guerre ! 19141918, Bruxelles, Casterman

\subsection{Sources secondaires}

Affolter, Michael (2011), Mündlichkeit im literarischen Dialog epischer Texte. Einführung des Konzepts der Mündlichkeitsintensität im Dienste der kontrastiven Linguistik und der literarischen Analyse, Stuttgart, Ibidem.

ATILF (1994), TLFi : Trésor de la langue Française informatisé, ATILF - CNRS \& Université de Lorraine [http://www.atilf.fr/tlfi.]. Baron-Carvais, Annie (2007), La bande dessinée, Paris, PUF.
Bauche, Henri (1920), Le langage populaire. Grammaire, syntaxe et dictionnaire du français tel qu'on le parle dans le peuple de Paris avec tous les termes d'argot usuel, Paris, Payot.

Bianchi, Nicolas (2015), " Mots du peuple et pratique de l'écrit », in Steuckardt, Agnès (éd.), Entre villages et tranchées. L'écriture de Poilus ordinaires, Uzès, Inclinaison, 125-142.

Blanche-Benveniste, Claire (1997), Approches de la langue parlée en français, Paris, Orphys (Collection L'essentiel français).

Blank, Andreas (1991), Literarisierung von Mündlichkeit: Louis-Fernand Céline und Raymond Queneau, Tübingen, Gunter Narr.

Bocklage, Katja / Otto, Nina (2018), « > Fusillés pour l'exemple «. Zur Darstellung des Ersten Weltkriegs im Werk von Jacques Tardi », in Junk, Claudia / Schneider, Thomas F. (éd.), Krieg in Comic, Graphic Novel und Literatur, Göttingen, V\&R unipress (Krieg und Literatur; 24), 25-44. 
Borcherding, Vivien / Mertins, Caroline / Riemer, Alexander (2018), «Abweichung von der Norm. Harvey Kurtzmans Frontline Combat (1951-1954) », in Junk, Claudia / Schneider, Thomas F. (ed.), Krieg in Comic, Graphic Novel und Literatur, Göttingen, V\&R unipress (Krieg und Literatur; 24), 7-24.

Bramlett, Frank (2016), "Comics and Linguistics " in Bramlett, Frank / Cook, Roy T. / Meskin, Aaron (éd.), The Routledge Companion to Comics, London, Routledge, 380-389.

Cohn, Neil (2012), «Comics, Linguistics, and Visual Language : The past and future of a field », in Bramlett, Frank (éd.), Linguistics and the study of comics, Basingstoke, Palgrave Macmillan, 92-118.

Dauzat, Albert (1919), L'argot de la guerre. D'après une enquête auprès des officiers et soldats, Paris, Armand Colin.

Déchelette, François (1972 [1918]), L'Argot des Poilus. Dictionnaire humoristique et philologique du langage des soldats de la Grande Guerre de 1914. Argots spéciaux des aviateurs, aérostiers automobilistes, etc, Genève, Slatkine Reprints.

Eisner, Will (1985), Comics and Sequential Art, Tamarac, FL, Poorhouse.

Elspaß, Stephan (2012), « The Use of Private Letters and Diaries in Sociolinguistic Investigation », in Hernández Campoy, Juan Manuel / Conde Silvestre, Juan Camilo (éd.), The Handbook of Historical Sociolinguistics, Malden, Mass et al., Wiley Blackwell (Blackwell Handbooks in Linguistics), 156-169.

Esnault, Gaston (1919), Le poilu tel qu'il se parle. Dictionnaire des termes populaires récents et neufs employés aux armées en 1914-1918 étudiés dans leur étymologie, leur développement et leur usage, Paris, Bossard.

Fludernik, Monika (2011), « Mündliches und schriftliches Erzählen », in Martínez, Matías (éd.), Handbuch Erzählliteratur. Theorie, Analyse, Geschichte, Stuttgart, Weimar, Metzler, 29-36.

François, Denise (1985), "Le langage populaire ", in Gérald, Antoine / Robert, Martin (éd.), Histoire de la langue française 18801914, Paris, CNRS, 295-327.

Gadet, Françoise (1992), Le français ordinaire, Paris, PUF.

Giaufret, Anna (2013), « Le français dans la bande dessinée québécoise : quelles représentations du français parlé ? ", in Repères DoRiF, 2, Voix/voies excentriques: la langue française face à l'altérité [http://www.dorif.it/ezine/ezine_printarticle.php?id=74].

Glaude, Benoît (2014), " Pour une approche littéraire des bandes dessinées ", in Bande dessinée et littérature, Neuvième art 2.0 [http://neuviemeart.citebd.org/spip.php?article805].

Goetsch, Paul (1985), « Fingierte Mündlichkeit in der Erzählkunst entwickelter Schriftkulturen », in Poética - Zeitschrift für Sprachund Literaturwissenschaft, 17, 202-218.

Große, Sybille / Sowada, Lena (2020), « Socialisation écrite et rédaction épistolaire de scripteurs moins expérimentés - lettres des soldats de la Grande Guerre », in Romanistisches Jahrbuch, 71(1), 82-129.

Große, Sybille / Steuckardt, Agnès / Sowada, Lena / Dal Bo, Beatrice (2016), « Du rituel à l'individuel dans des correspondances peu lettrées de la Grande Guerre », in SHS Web of Conferences, 27, Congrès Mondial de Linguistique Française - CMLF 2016, 1-15 [DOI : 10.1051/shsconf/20162706008].

Guilhaumou, Jacques (1996), « Vers une histoire des événements linguistiques. Un nouveau protocole d'accord entre I'historien et le linguiste », in Histoire Épistémologie Langage 18 (2), 103-126.
Hennig, Mathilde / Jacob, Joachim (2016), « Nähe, Distanz und Literatur », in Feilke, Helmuth / Mathilde, Hennig (éd.), Zur Karriere von ,Nähe und Distanz'. Rezeption und Diskussion des Koch-Oesterreicher-Modells, Berlin, Boston, De Gruyter (Germanistische Linguistik, 306), 187-211.

Huot, Hélène (2005), La morphologie. Forme et sens des mots du français, Paris, Armand Colin (Collection Cursus / Linguistique). Koch, Peter / Oesterreicher, Wulf (1986), « Sprache der Nähe - Sprache der Distanz. Mündlichkeit und Schriftlichkeit im Spannungsfeld von Sprachtheorie und Sprachgeschichte ", in Romanistisches Jahrbuch 1985(36), 15-43.

Koch, Peter / Oesterreicher, Wulf (2011), Gesprochene Sprache in der Romania. Französisch, Italienisch, Spanisch, Berlin, De Gruyter (Romanistische Arbeitshefte, 31).

Krieger, Swetlana / Machnik, Julia / Schmitz, Wiebke (2018), « Tagebuch 14/18 - Vier Geschichten aus Deutschland und Frankreich.

Ein Beitrag zur transnationalen Erinnerungskultur im Kontext des 100. Jahrestags des Ersten Weltkriegs », in Junk, Claudia / Schneider, Thomas F. (éd.), Krieg in Comic, Graphic Novel und Literatur, Göttingen, V\&R unipress (Krieg und Literatur ; 24), 45-59.

Littré, Émile (1873-1874), Dictionnaire de la langue française, Paris, L. Hachette.

Mahrer, Rudolf (2017), Phonographie. La représentation écrite de l'oral en français, Berlin, Boston, De Gruyter (Études de linguistique française ; 3 ).

McCloud, Scott (1993), Understanding Comics : The Invisible Art, Northampton, MA, Kitchen Siuk.

Mercier, Simon (2015), «Dans la Grande Histoire », in Steuckardt, Agnès (éd.), Entre villages et tranchées. L'écriture de Poilus ordinaires, Uzès, Inclinaison, 19-39.

Meskin, Aaron (2016), « Defining comics », in Bramlett, Frank / Cook Roy T. / Meskin, Aaron (éd.), The Routledge Companion to Comics, London, Routledge, 221-229.

Müller, Bodo (1975), Das Französische der Gegenwart. Varietäten Strukturen - Tendenzen, Heidelberg, Winter.

Paulikat, Frank (2017), Wortbildung des heutigen Französisch, Berlin, Boston, De Gruyter (Romanistische Arbeitshefte ; 66).

Pietrini, Daniela (2007), Parola di papero. Storia e tecniche della lingua dei fumetti Disney, Floerence, Franco Cesati Editore.

Poirier, Claude / Francard, Michel (2019), Base de données lexicographiques panfrancophone (BDLP) [http://www.bdlp.org/].

Prüßmann-Zemper, Helga (1990), « Französisch : Varietätenlinguistik des Französischen », in Holtus, Günter / Metzeltin, Michael / Schmitt, Christian (éd.), Französisch. Le français, Tübingen, Niemeyer (Lexikon der Romanistischen Linguistik (LRL), V, 1), 830-843.

Quinquis, Stella (2004), Die literarisch konstruierte Mündlichkeit in "Les Frustrés » von Claire Brétecher, Dissertationsschrift, Bochum, Universität Bochum.

Rey, Alain (2010), Dictionnaire historique de la langue française, Paris, Le Robert.

Rézeau, Pierre (2018), Les mots des Poilus dans leurs correspondances et leurs carnets, Strasbourg, ELIPHI.

Rifas, Leonard (2016), "War Comics », in Bramlett, Frank / Cook, Roy T. / Meskin, Aaron (éd.), The Routledge Companion to Comics, London, Routledge, 183-191.

Sainéan, Lazare (1915), L'Argot des Tranchées d'après les Lettres des Poilus et les Journaux du Front, Paris, De Boccard. 
Schiegg, Markus / Sowada, Lena (2019), « Script Switching in 19th-Century Lower-Class German Handwriting », in Paedagogica Historica 55/6, 2019, 772-791.

Schlieben-Lange, Brigitte (1983), Traditionen des Sprechens. Elemente einer pragmatischen Sprachgeschichtsschreibung, Stuttgart, Kohlhammer.

Sinner, Carsten (2014), Varietätenlinguistik. Eine Einführung, Tübingen, Narr (Narr-Studienbücher).

Sowada, Lena (2018), " La correspondance privée des gens ordinaires : l'expression écrite entre langue de l'immédiat communicatif et traditions discursives ", in Cahiers de praxématique, 71 [https:// doi.org/10.4000/praxematique.4991, consulté le 01.12.2020].

Steuckardt, Agnès (2018), "Les corpus peu lettrés de la Grande Guerre en linguistique française : de la grammaire à la textométrie ", in Steffen, Joachim / Thun Harald / Zaiser Rainer (éd.), Classes populaires, scripturalité et histoire de la langue. Un bilan interdisciplinaire, Kiel, Westensee-Verlag (Dialectologia pluridimensionalis romanica, 17), 25-47.

Steuckardt, Agnès / Große, Sybille / Dal Bo, Beatrice / Sowada, Lena (2020), « Le rituel et l'individuel dans les pratiques d'écriture. L'exemple des formules de clôture dans des correspondances peu lettrées de la Grande Guerre », in Remysen, Wim / Tailleur, Sandrine (éd.), L'individu et sa langue. Hommages à France Martineau, Québec, Presses de I'Université Laval (Les Voies du français).

Thun, Harald (2005), « Literarisierte Mündlichkeit und Sprachwandel ", in Pusch, Claus D. / Kabatek, Johannes / Raible, Wolfgang (éd.), Romanistische Korpuslinguistik II. Romance Corpus Linguistics II. Korpora und diachrone Sprachwissenschaft / Corpora and Diachronic Linguistics, Tübingen, Narr, 85-108.

Tuaillon, Gaston (1978), "Régionalismes de France ", in Revue de Linguistique Romane, 42, 149-194. 